\title{
Rapid Fading Due to Human Blockage in Pedestrian Crowds at 5G Millimeter-Wave Frequencies
}

\author{
George R. MacCartney Jr., Theodore S. Rappaport, and Sundeep Rangan \\ NYU WIRELESS \\ NYU Tandon School of Engineering \\ New York University, Brooklyn, NY 11201 \\ $\{$ gmac,tsr,srangan $\} @$ nyu.edu
}

\begin{abstract}
Rapidly fading channels caused by pedestrians in dense urban environments will have a significant impact on millimeter-wave (mmWave) communications systems that employ electrically-steerable and narrow beamwidth antenna arrays. A peer-to-peer (P2P) measurement campaign was conducted with $7^{\circ}, 15^{\circ}$, and $60^{\circ}$ half-power beamwidth (HPBW) antenna pairs at $73.5 \mathrm{GHz}$ and with $1 \mathrm{GHz}$ of RF null-to-null bandwidth in a heavily populated open square scenario in Brooklyn, New York, to study blockage events caused by typical pedestrian traffic. Antenna beamwidths that range approximately an order of magnitude were selected to gain knowledge of fading events for antennas with different beamwidths since antenna patterns for mmWave systems will be electronically-adjustable. Two simple modeling approaches in the literature are introduced to characterize the blockage events by either a two-state Markov model or a four-state piecewise linear modeling approach. Transition probability rates are determined from the measurements and it is shown that average fade durations with a $-3 \mathrm{~dB}$ threshold are $299.0 \mathrm{~ms}$ for $7^{\circ}$ HPBW antennas and $260.2 \mathrm{~ms}$ for $60^{\circ} \mathrm{HPBW}$ antennas. The four-state piecewise linear modeling approach shows that signal strength decay and rise times are asymmetric for blockage events and that mean signal attenuations (average fade depths) are inversely related to antenna HPBW, where $7^{\circ}$ and $60^{\circ} \mathrm{HPBW}$ antennas resulted in mean signal fades of $15.8 \mathrm{~dB}$ and $11.5 \mathrm{~dB}$, respectively. The models presented herein are valuable for extending statistical channel models at mmWave to accurately simulate real-world pedestrian blockage events when designing fifth-generation (5G) wireless systems.
\end{abstract}

Index Terms-Millimeter-wave, 5G, mmWave, channel sounder, $73 \mathrm{GHz}$, real-time, direct-correlation, dynamic, fading, blockage, pedestrians, crowds.

\section{INTRODUCTION}

Millimeter-wave (mmWave) frequencies will play an important role in fifth-generation $(5 \mathrm{G})$ communications systems that are predicted to deliver gigabit data throughput speeds to mobile devices [1], [2]. Large-scale propagation characteristics of mmWave bands have been heavily studied in recent years to generate channel models and simulators for $5 \mathrm{G}$ system and network design [3]-[6]. However, few outdoor measurements across the mmWave bands have been conducted to understand channel dynamics and fading [7], [8]. MmWave signals are highly susceptible to blockage which leads to rapid channel variations that have an impact across the protocol stack [9].

Some indoor studies have been performed for rapid fading caused by human blockage events, particularly at $60 \mathrm{GHz}[10]-$ [13]. Early human blockage models were developed for the

This material is based upon work supported by NOKIA and the NYU WIRELESS Industrial Affiliates Program, three National Science Foundation (NSF) Research Grants: 1320472, 1302336, and 1555332, and the GAANN Fellowship Program. The authors thank S. Sun, Y. Xing, H. Yan, J. Koka, R. Wang, and D. Yu, for their help in conducting the measurements. 802.11ad standard which is meant for indoor short-range and high throughput applications [14]. Measurements by Collonge et al. showed that a single person shadowing the direct path between the transmitter (TX) and receiver (RX) would cause more than $20 \mathrm{~dB}$ of attenuation for approximately 100 milliseconds (ms), whereas large groups of 11-15 people can cause fade durations of $300 \mathrm{~ms}$ or more [10]. Short-range office measurements by Jacob et al. demonstrated that fade durations at $60 \mathrm{GHz}$ in line-of-sight (LOS) environments were on average $550 \mathrm{~ms}$ with mean attenuations between $6 \mathrm{~dB}$ and $18 \mathrm{~dB}$ [11].

Outdoor measurements in a street canyon by Weiler et al. using omnidirectional antennas at $60 \mathrm{GHz}$ indicated that attenuation could exceed $40 \mathrm{~dB}$ when the LOS path is blocked [15]. In the event of human body shadowing, the LOS multipath component (MPC) experienced extreme fading (more than 20 $\mathrm{dB}$ ), while secondary reflected MPCs were not affected [15]. An experiment at $28 \mathrm{GHz}$ with omnidirectional antennas in an urban open square studied shadowing caused by pedestrians and vehicles for a small cell, base station-to-mobile scenario [16]. Results indicated $12 \mathrm{~dB}$ to $25 \mathrm{~dB}$ attenuation of the LOS path for various shadowing events and a double knife-edge diffraction model exhibited a good approximation to human body blockage observations, similar to work in [13], [17], [18].

Work in [19] and [20] showed that fade depths decreased as bandwidth increased, and plateaued at bandwidths of $1 \mathrm{GHz}$ with rapid fading of $4 \mathrm{~dB}$. Similar observations were made in [7] at the $73 \mathrm{GHz}$ mmWave band in an urban setting, and spawn the motivation for studying the relationship between antenna beamwidth and signal bandwidth for fading effects caused by human blockage. These studies, however, did not provide a precise statistical characterization of the blockage dynamics that can be used for simulations. This work focuses on rapid fading caused by pedestrians in crowded urban scenarios. We report peer-to-peer (P2P) measurements across a busy walkway in a downtown open square and perform simple statistical techniques to model the results. Section II describes the channel sounder and measurement setup and environment. Section III describes two simple models used for analyzing the measurement results. Section IV provides the results and analysis and concluding remarks are made in Section V.

\section{Measurement Setup and Hardware}

The measurements were designed to study the effects of large moving crowds in an urban setting for a peer-to-peer communications links at mmWave. The measurement location is an open square in downtown Brooklyn, New York at the MetroTech Commons courtyard, which is surrounded on all 


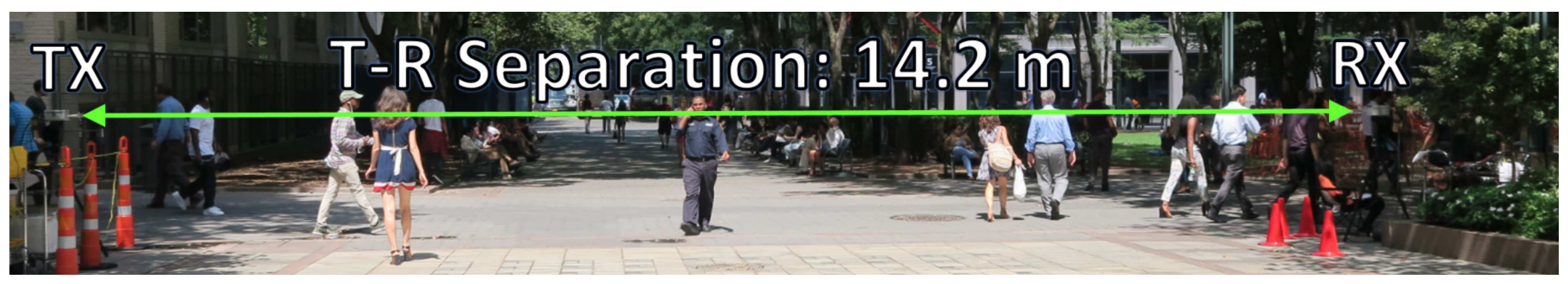

Fig. 1: Photo of test environment showing the intersection of Lawrence Street and Myrtle Avenue in downtown Brooklyn, New York.

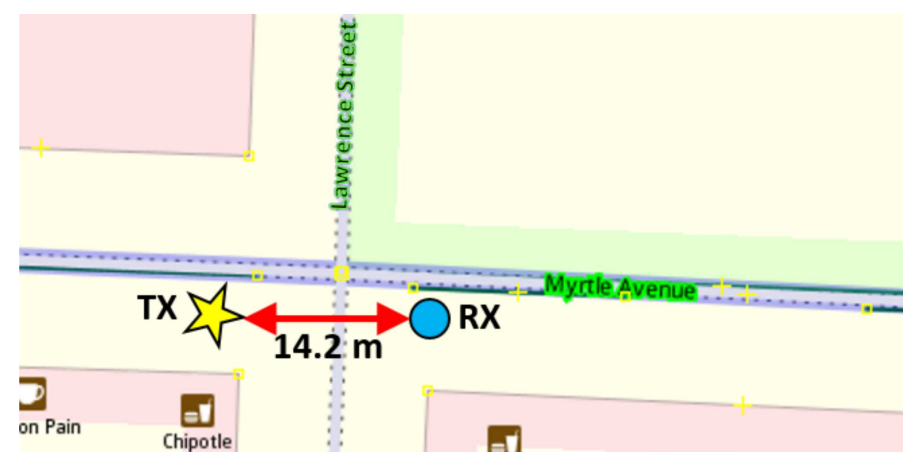

Fig. 2: Map of measurement environment in downtown Brooklyn, New York. The yellow star is the TX location and the blue circle is the RX location, with a T-R separation distance of $14.2 \mathrm{~m}$. A majority of traffic moved perpendicular to the line drawn between the TX and RX along Lawrence Street.

TABLE I: Descriptions of the three measurement tests.

\begin{tabular}{|c|c|c|c|}
\hline Measurement \# & Test 1 & Test 2 & Test 3 \\
\hline TX \& RX antenna gain & $27 \mathrm{dBi}$ & $20 \mathrm{dBi}$ & $9.1 \mathrm{dBi}$ \\
\hline TX \& RX Az/El HPBW & $7^{\circ} / 7^{\circ}$ & $15^{\circ} / 15^{\circ}$ & $60^{\circ} / 60^{\circ}$ \\
\hline T-R separation & \multicolumn{3}{|c|}{$14.2 \mathrm{~m}$} \\
\hline Observation window & \multicolumn{3}{|c|}{$135 \mathrm{~s}$} \\
\hline Total PDPs recorded & \multicolumn{3}{|c|}{40,800} \\
\hline PDP time/frequency interval & \multicolumn{3}{|c|}{$\sim 3.3 \mathrm{~ms} / 302 \mathrm{~Hz}$} \\
\hline Carrier frequency & \multicolumn{3}{|c|}{$73.5 \mathrm{GHz}$} \\
\hline RF null-to-null bandwidth & \multicolumn{3}{|c|}{$1 \mathrm{GHz}$} \\
\hline Pedestrian/bicyclist crossings per minute & 17.8 & 12.9 & 12 \\
\hline
\end{tabular}

four sides by typical office skyscrapers and the New York University Tandon School of Engineering campus buildings. The measurements were purposely conducted in a high foot-traffic area where pedestrians, child strollers, and bicyclists typically traverse in large quantities. Fig. 2 shows a map layout of the measurement area where the TX and RX are located on opposite sides of the walkway and were set at heights of $1.5 \mathrm{~m}$ relative to the ground, with a transmitter-receiver (T-R) separation distance of $14.2 \mathrm{~m}$. Fig. 1 shows a photo of the test scenario. Table I specifies the number of pedestrians or bicyclists that passed through the link per minute for each test. The pedestrians were never closer than $1.5 \mathrm{~m}$ from the TX or RX antennas and on average were equidistant from the TX and RX.

Three measurement tests were performed with different pairs of TX and RX horn antennas in order to study the relationship between rapid fading shadowing events and antenna beamwidth. Table I provides the measurement specifications for each test with $7^{\circ}, 15^{\circ}$, and $60^{\circ}$ azimuth (Az) and elevation (El) HPBW antenna pairs used for test one, test two, and test three, respectively. Three different antenna beamwidth pairs that range approximately an order of magnitude difference were used to gain insights into fading events in pedestrian crossings at mmWave, especially since various phased-array architectures with flexible antenna patterns are expected for base stations and mobile handsets. It is also expected that the largest antenna beamwidth for mmWave systems will be no more than $60^{\circ}$ which is why it was the maximum beamwidth used for this experiment [7]. For each test, the antennas were boresightaligned and remained fixed. Each test consisted of a 135-second free-running observation window in which power delay profiles (PDPs) were captured with a $\sim 302 \mathrm{~Hz}$ snapshot interval. Each observation window resulted in 40,800 PDP snapshots with a $\sim 3.3 \mathrm{~ms}$ time separation between back-to-back PDPs. A -20 dB max peak threshold was applied to each PDP in post-processing and the area under each of the PDPs was integrated to calculate the total received power for every snapshot. We note that due to spatial filtering by the directional antennas, only one multipath cluster (LOS path) was detectable over all measurements.

The real-time spread spectrum correlator (direct-correlation) channel sounder described in [21] was used for the measurements at a $73.5 \mathrm{GHz}$ carrier frequency with $1 \mathrm{GHz}$ of $\mathrm{RF}$ null-to-null bandwidth and a 2 ns MPC time resolution. True propagation delay timing was calibrated as described in [21]. The maximum effective isotropic radiated power (EIRP) was $21.2 \mathrm{dBm}$ when the channel sounder was configured with a TX output power of $-5.8 \mathrm{dBm}$ and with the $27 \mathrm{dBi}$ gain and $7^{\circ} \mathrm{Az} / \mathrm{El}$ HPBW TX antenna. Additional details for the channel sounder are provided in [21].

\section{Modeling Methodology}

Of many potential modeling strategies, two simple methodologies are used here to model the blockage events: 1) a two-state approach with unshadowed and shadowed states; 2) a four-state approach with unshadowed, decaying signal level, shadowed, and rising signal level states.

\section{A. Two-State Blockage Modeling}

A simple two-state Markov model can be used to characterize unshadowed and shadowed states for a wireless link in the presence of pedestrian induced variations in received signal strength [22], [23]. Fig. 3 shows a diagram of a two-state Markov model where $P_{\text {unshad }}$ and $P_{\text {shad }}$ indicate the transition probabilities of going from a shadowed to unshadowed state and an unshadowed to shadowed state, respectively. A predefined threshold is selected in order to characterize a shadowed or unshadowed state and is typically $0 \mathrm{~dB}$ to $-10 \mathrm{~dB}$ from the zerocrossings just before and after the fading event. Fig. 4 depicts the characterization of a typical blockage event with two-states when applying a $0 \mathrm{~dB}$ threshold relative to the zero-crossings for the beginning and end of a shadowing event. 


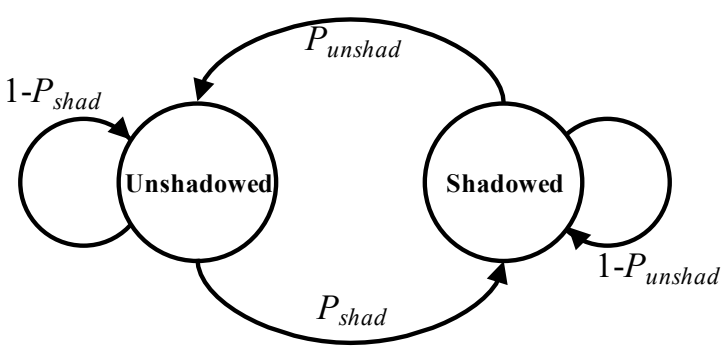

Fig. 3: Two-state Markov model for blockage events.

Two-State Pedestrian Blockage Event

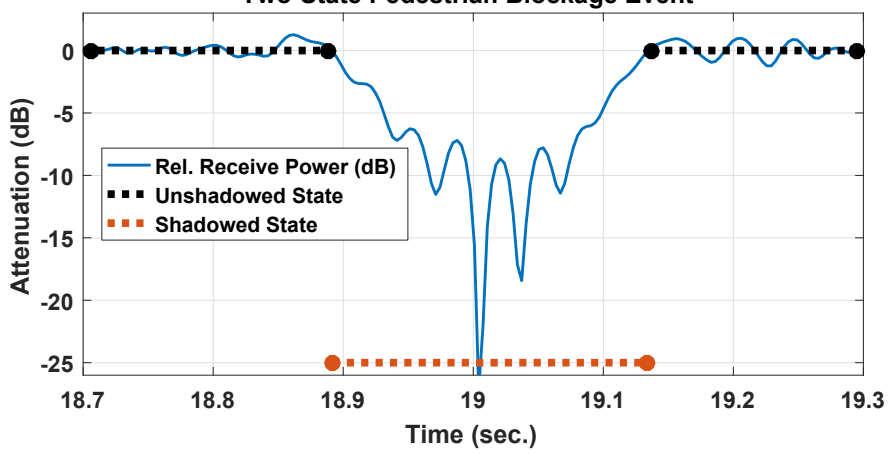

Fig. 4: Two-state shadowing event with $0 \mathrm{~dB}$ threshold showing unshadowed (black line) and shadowed (red line) regions

\section{B. Four-State Blockage Modeling}

A four-state piecewise linear modeling approach is also useful for characterizing blockage with the following regions: unshadowed, shadowed, a decaying signal level region from unshadowed to shadowed, and a rising signal level region from shadowed to unshadowed [11]. The blockage event signal level (in $\mathrm{dB}$ ) as a function of time, $S E(t)$ is represented as [11], [17]:

$$
S E(t)[\mathrm{dB}]= \begin{cases}r_{\text {decay }} \cdot t, & \text { for } 0 \leq t \leq \frac{S E_{\text {mean }}}{r_{\text {decay }}} \\ S E_{\text {mean }}, & \text { for } \frac{S E_{\text {mana }}}{r_{\text {decay }}} \leq t \leq t_{D}-\frac{S E_{\text {mean }}}{r_{\text {rise }}} \\ S E_{\text {mean }}-r_{\text {rise }} \cdot t, & \text { for } t_{D}-\frac{S E_{\text {mean }}}{r_{\text {rise }}} \leq t \leq t_{D} \\ 0, & \text { otherwise }\end{cases}
$$

where $r_{\text {decay }}$ is the signal strength decay rate in $\mathrm{dB} / \mathrm{ms}, t$ is the time in $\mathrm{ms}$ from the onset $(t=0 \mathrm{~ms})$ of the blockage event, $S E_{\text {mean }}$ is the blockage event mean signal attenuation in $\mathrm{dB}$, $r_{\text {rise }}$ is the blockage event signal strength rise rate in $\mathrm{dB} / \mathrm{ms}$, and $t_{D}$ is the shadowing event fade time duration in ms [24]. The $r_{\text {decay }}$ and $r_{\text {rise }}$ rates are determined by the decay time $t_{\text {decay }}$, rise time $t_{\text {rise }}$, and either a predetermined threshold between the last zero-crossing before and the first zero-crossing after the rapid signal fade, or is strictly based on the mean signal attenuation of the shadowing event $S E_{\text {mean }}$, calculated over the interval $\left[\frac{1}{3} t_{D}<\right.$ $\left.t<\frac{2}{3} t_{D}\right]$. Regardless of the threshold method used, the blockage event mean signal attenuation $S E_{\text {mean }}$ is determined over the same interval. Fig. 5 shows an example of a blockage event with a threshold of $S E_{\text {mean }}$.

The selected threshold level will affect the decay and rise times of the blockage events, and by consequence, will influence the decay and rise rates of signal strength. Therefore, blockage modeling for different applications may require using various thresholds depending upon the system requirements and fade margin limitations. For example, the blockage events in Fig. 6 were determined with a $-5 \mathrm{~dB}$ threshold and the unshadowed,

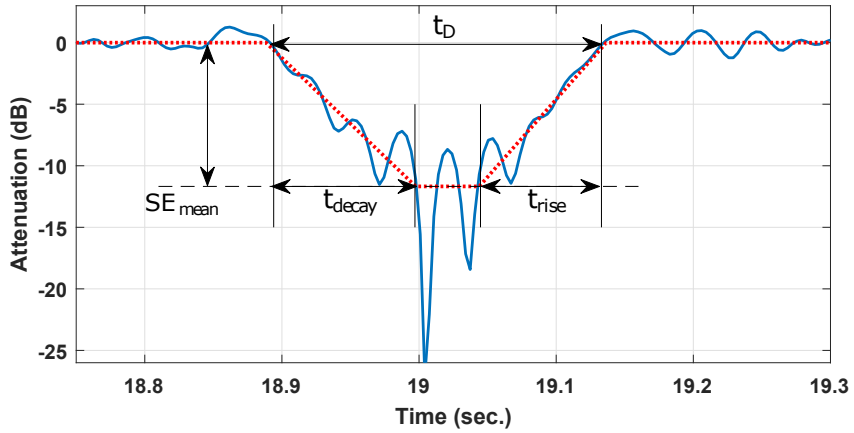

Fig. 5: Four-state shadowing event with an $S E_{\text {mean }}$ calculated threshold and labels for decay time $t_{\text {decay }}$, rise time $t_{\text {rise }}$, shadowing event mean attenuation $S E_{\text {mean }}$, and fade duration $t_{D}$.

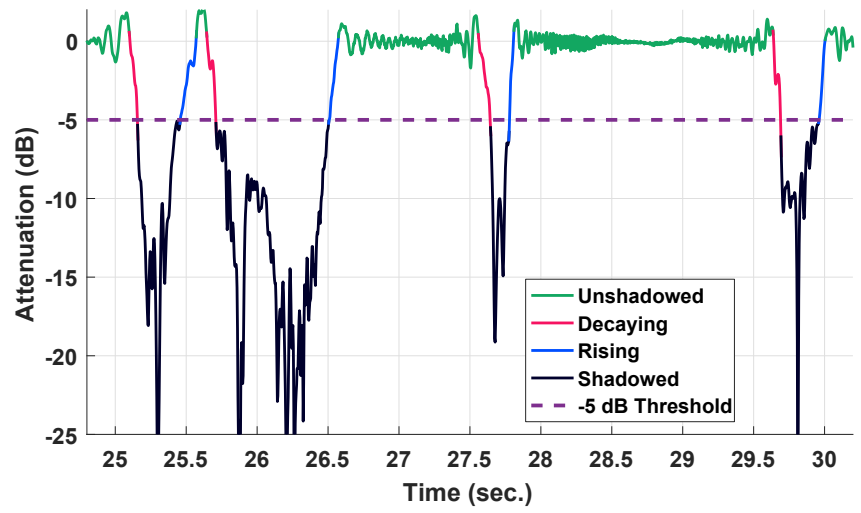

Fig. 6: Four-state piecewise linear model with unshadowed, decaying, shadowed, and rising regions during a $73.5 \mathrm{GHz}$ blockage event with a $-5 \mathrm{~dB}$ pre-defined threshold, as an example.

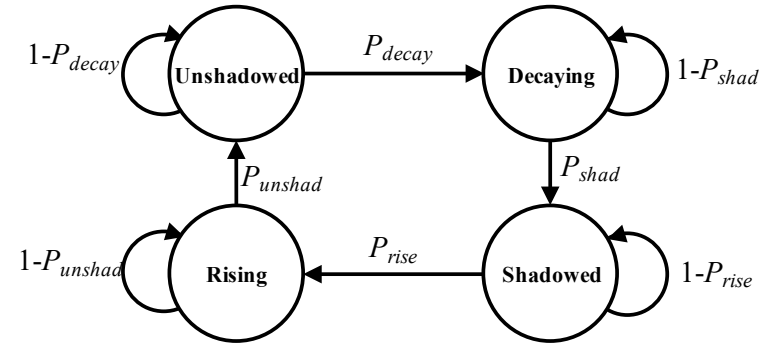

Fig. 7: Four-state Markov model for unshadowed, decaying, shadowed, and rising regions for modeling blockage events.

decaying, shadowed, and rising regions are indicated by different colors.

The example in Fig. 6 brings about a four-state Markov model shown in the diagram in Fig. 7. The transition probabilities in Fig. 7 are defined as: $P_{\text {decay }}$ is the probability of transitioning from an unshadowed state to a state of decaying signal levels, $P_{\text {shad }}$ is the probability of transitioning from a state of decaying signal levels to a shadowed state, $P_{\text {rise }}$ is the probability of transitioning from a shadowed state to a rising signal level state, and $P_{\text {unshad }}$ is the probability of transitioning from a rising signal state to an unshadowed/unblocked state.

\section{Measurement Results and Analysis}

\section{A. Two-State Blockage Model}

While various thresholds could be selected to define the start of a shadowing event, a $-3 \mathrm{~dB}$ threshold from the unshadowed 
$0 \mathrm{~dB}$ attenuation level was used to compare the blockage observations for different antenna pairs. Note that blockage events were initially labeled using the Lloyd-Max algorithm (2 states) in MATLAB, but were analyzed and modeled using the criteria and threshold defined here. Table II provides the transition probability rate $\lambda$ values of conditional state transitions for the observed blockage events. For a transition probability $p$ and a sampling interval $T, \lambda=p / T$. For example, the probability of transitioning from a shadowed state to an unshadowed state with $7^{\circ} \mathrm{HPBW}$ antennas is $p=0.0007$ for the sampling interval $T=3.3 \mathrm{~ms}$, such that $\lambda=0.21$ transitions $/$ second (trans $/ \mathrm{sec}$ ). In Table II, $\lambda$ represents the conditional probability rate for transitioning from a "current state" to the "next state."

The transition rates for a shadowed state conditioned on an unshadowed state are consistent and around 0.20 trans/sec for the three antenna HPBW pairs and for all antenna tests combined. These transition rates imply that there are approximately $5 \mathrm{sec}-$ onds between shadowed states. The transition rates are slightly different for an unshadowed state conditioned on a shadowed state, where the rate is correlated with the antenna HPBW. For instance, $\lambda$ is 3.36 and 3.85 for $7^{\circ}$ and $60^{\circ}$ HPBW TX/RX antennas, respectively, indicating that blockage events are longer for narrower beamwidth antennas $\left(7^{\circ}: 298 \mathrm{~ms}\right)$ compared with wider beamwidth antennas $\left(60^{\circ}: 260 \mathrm{~ms}\right)$. This intuitively makes sense since wider beamwidth antennas would result in more energy spread around the obstructions from the transmitter, and a wider viewing angle at the receiver to capture diffracted energy around the pedestrian blockers.

Fig. 8 shows the cumulative distribution function (CDF) curves of the pedestrian blockage event fade time durations for each set of antennas and when blockage event observations from all antenna sets are considered together for analysis. Table III provides the best-fit CDF parameters* ${ }^{*}$ to the empirical CDF data in Fig. 8 (using the fitdist function in MATLAB ${ }^{\circledR}$ ) and their goodness of fit (GOF) to the data (using the goodnessOfFit function in MATLAB ${ }^{\circledR}$ ) where the normalized mean square error (NMSE) GOF measure ranges from $-\infty$ to 1 , where $-\infty$ indicates a poor fit, and 1 indicates a perfect fit [25]. Similar to the transition probability rates, the mean fade time duration reduces as antenna beamwidth increases. For example, the mean fade time durations are $299.0 \mathrm{~ms}, 267.4 \mathrm{~ms}$, and $260.2 \mathrm{~ms}$ for $7^{\circ}, 15^{\circ}$, and $60^{\circ} \mathrm{TX} / \mathrm{RX}$ HPBW antenna pairs, respectively. The GOF values for the CDFs in Table III are all 0.95 or above and indicate a good match to the empirical data.

While 100's of milliseconds for a fade duration may seem short, it is significant for wireless communications systems. For instance, the 802.11ad standard for $60 \mathrm{GHz}$ has packet

TABLE II: Two-state Markov model transition probability rates for human blockage events at $73.5 \mathrm{GHz}$.

\begin{tabular}{|c|c|c|c|}
\hline TX/RX HPBW & Current State & Next State & $\lambda$-rate $($ trans/sec) \\
\hline \multirow{2}{*}{$7^{\circ}$} & unshadowed & shadowed & 0.21 \\
\cline { 2 - 4 } & shadowed & unshadowed & 3.36 \\
\hline \multirow{2}{*}{$15^{\circ}$} & unshadowed & shadowed & 0.21 \\
\cline { 2 - 4 } & shadowed & unshadowed & 3.42 \\
\hline \multirow{2}{*}{$60^{\circ}$} & unshadowed & shadowed & 0.18 \\
\cline { 2 - 4 } & shadowed & unshadowed & 3.85 \\
\hline \multirow{2}{*}{ All Combined } & unshadowed & shadowed & 0.18 \\
\cline { 2 - 4 } & shadowed & unshadowed & 3.52 \\
\hline
\end{tabular}

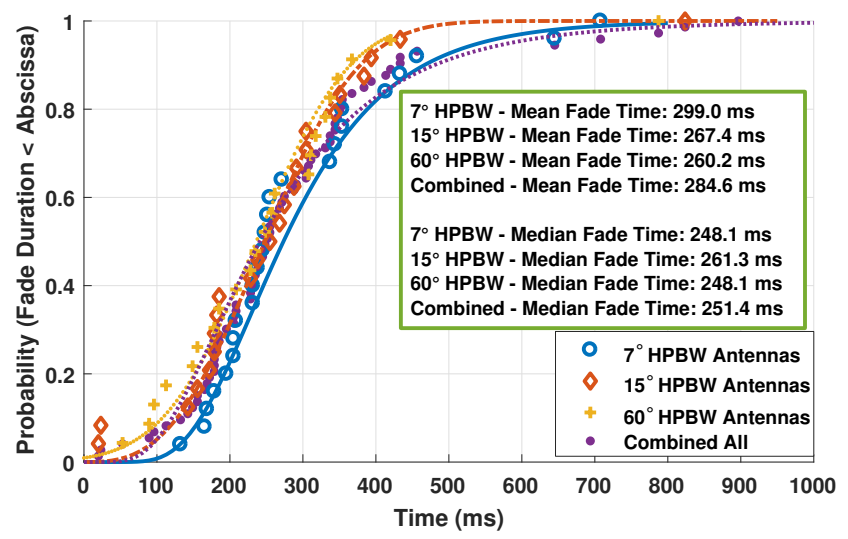

Fig. 8: Two-state Markov model mean fade time duration (ms) CDFs for the $73.5 \mathrm{GHz}$ blockage measurements. The lines in the plot represent the CDF curves best-fit to the data with parameters provided in Table III.

TABLE III: CDF parameters for the two-state Markov model.

\begin{tabular}{|c|c|c|c|}
\hline \multicolumn{4}{|c|}{ CDF Parameters for Fade Duration [ms] of Two-State Markov Model } \\
\hline Ant. HPBW & Dist. & Parameters & GOF \\
\hline $7^{\circ}$ & Log-normal & $\mu=5.61 ; \sigma=0.42$ & 0.95 \\
\hline $15^{\circ}$ & Weibull & $\alpha=282.12 ; \beta=2.84$ & 0.98 \\
\hline $60^{\circ}$ & Normal & $\mu=236.22 ; \sigma=100.50$ & 0.98 \\
\hline $7^{\circ} ; 15^{\circ} ; 60^{\circ}$ & Log-normal & $\mu=5.48 ; \sigma=0.54$ & 0.98 \\
\hline
\end{tabular}

transmission times on the microsecond level and thus a blockage event of $200 \mathrm{~ms}$ to $300 \mathrm{~ms}$ would result in severe throughput degradation or an outage over that time span. Therefore, access point diversity with multiple nodes can be coordinated in MAC protocols to maintain a reliable connection to a mobile or user equipment (UE) [26]. Additionally, electrically-steerable antennas envisioned for mmWave communications can be used to beam switch around obstacles in order to find reflections and scatterers that avoid pedestrian blockages.

\section{B. Four-State Blockage Model}

The piecewise linear model described in Section III-B was used to model four states of blockage events: unshadowed, decaying signal strength, shadowed, and rising signal strength. Similar to the two-state model in Section IV-A, blockage events were initially labeled using the Lloyd-Max algorithm in MAT$\mathrm{LAB}$, but were analyzed and modeled using the criteria and threshold defined here. For the results shown here, a $0 \mathrm{~dB}$ threshold for the first and last zero-crossings of the blockage event was used to determine the beginning and end times of the decaying and rising states, respectively [11], [17]. The mean attenuation level calculated over the interval $\left[\frac{1}{3} t_{D}<t_{D}<\frac{2}{3} t_{D}\right]$ was used as the threshold for determining the shadowed state. Following this procedure, the shadowing event fade time durations $t_{D}$, decaying signal strength rates $r_{\text {decay }}$, the mean attenuation of shadowing events $S E_{\text {mean }}$, and rising signal strength rates $r_{\text {rise }}$ were determined for each individual blockage event of the measurement tests.

Fig. 9a shows the CDFs of the signal strength decay rates in $\mathrm{dB} / \mathrm{ms}$ for the three TX/RX antenna HPBW pairs along with the best-fit CDF curves* to the empirical data and with best-fit

\footnotetext{
${ }^{*}$ Note that all best-fit CDFs were derived using the fitdist function and all GOF values were calculated using the goodnessOfFit function, both in MATLAB ${ }^{\circledR}$. We also note that due to the limited measured data, the last few percent of data of each of the tails was ignored when solving for the best-fit distributions.
} 


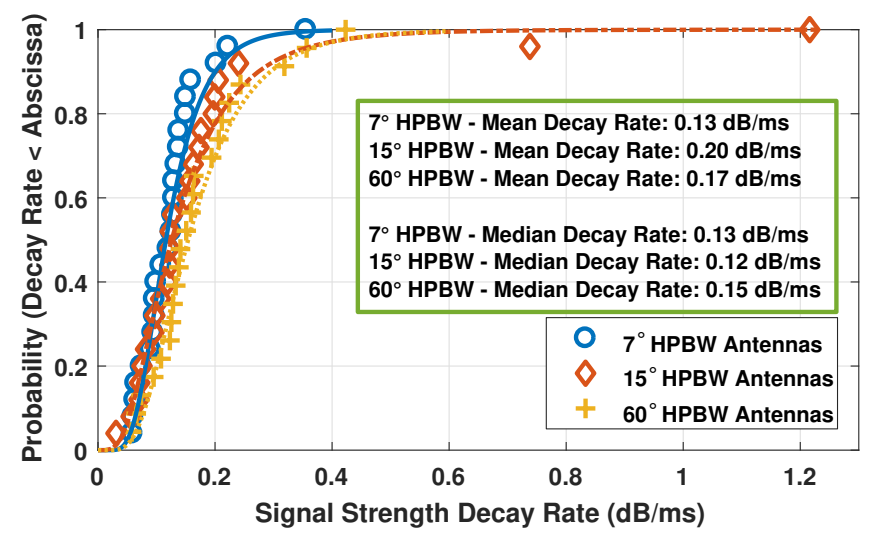

(a) Signal strength decay rate $r_{\text {decay }}(\mathrm{dB} / \mathrm{ms}) \mathrm{CDFs}$

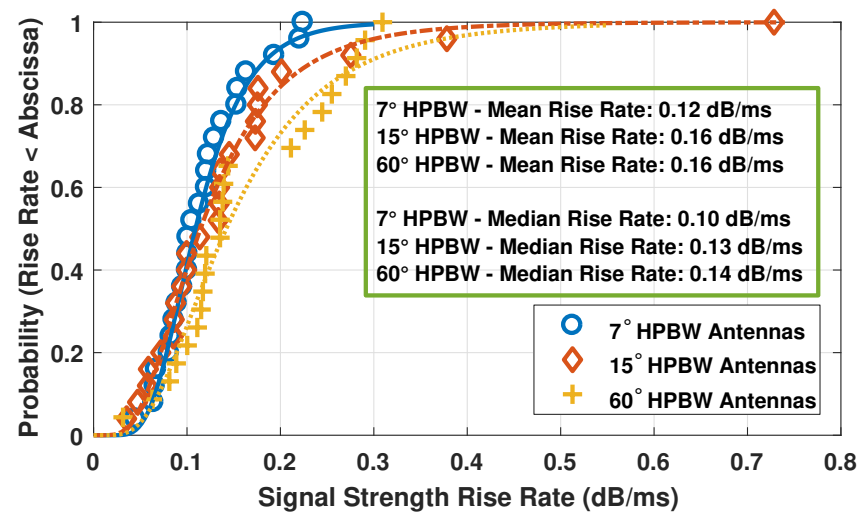

(b) Signal strength rise rate $r_{\text {rise }}(\mathrm{dB} / \mathrm{ms}) \mathrm{CDFs}$

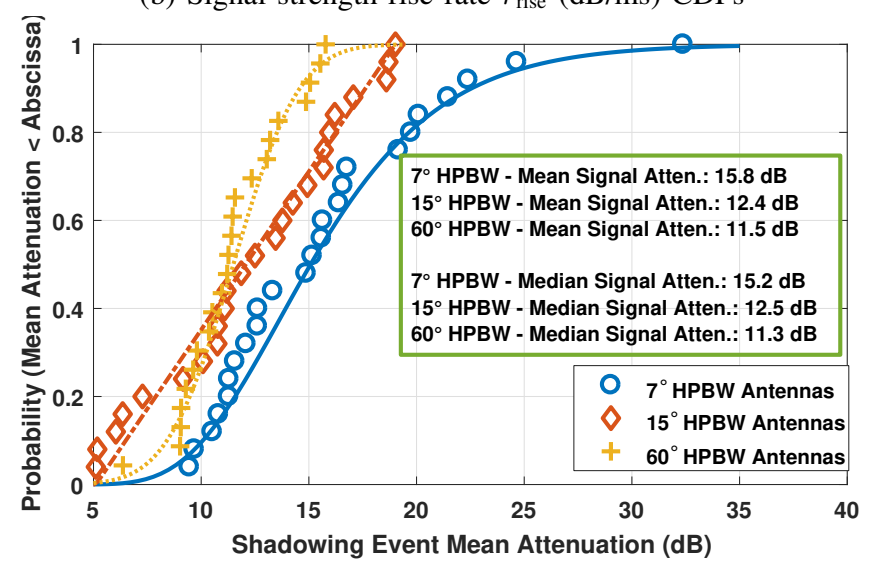

(c) Shadowing event mean attenuation $S E_{\text {mean }}(\mathrm{dB}) \mathrm{CDFs}$

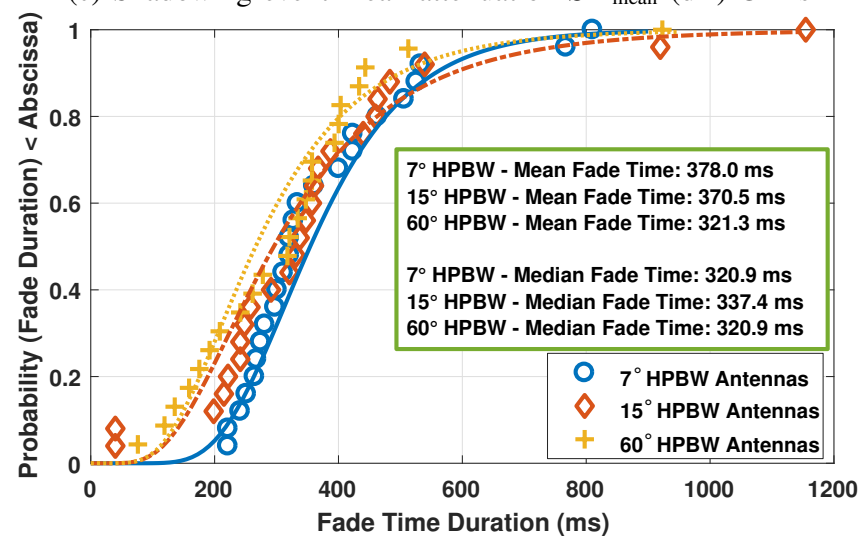

(d) Fade time duration $t_{D}(\mathrm{~ms}) \mathrm{CDFs}$

Fig. 9: Four-state piecewise linear blockage model CDFs for decay rate, rise rate, mean signal attenuation, and fade time duration for each pair of HPBW antennas at $73.5 \mathrm{GHz}$. The lines in (a) to (d) represent the CDF curves best-fit to the data with parameters provided in Table IV. model parameters provided in Table IV. The GOF values are all 0.93 or above, indicating a good distribution fit to the data. The signal strength decay rates are determined as the rate of change over the time-frame $\left(t_{\text {decay }}\right)$ from the last zero-crossing before the fading event and the first instance of the signal level at or below the mean attenuation value. The median values for the signal strength decay rates give a better indication of the trend than the mean values since the $15^{\circ} \mathrm{HPBW}$ antenna tests had two extremely rapid decay rates of $0.74 \mathrm{~dB} / \mathrm{ms}$ and $1.22 \mathrm{~dB} / \mathrm{ms}$ when a hand waved two times between the LOS link, which skewed the mean decay rate. The median decay rates of $0.13 \mathrm{~dB} / \mathrm{ms}$ and $0.12 \mathrm{~dB} / \mathrm{ms}$ for the $7^{\circ}$ and $15^{\circ} \mathrm{HPBW}$ antennas, respectively, show that relatively narrowbeam TX and RX antennas result in similar blockage decay rates. However, the median decay rate of $0.15 \mathrm{~dB} / \mathrm{ms}$ for the $60^{\circ} \mathrm{HPBW}$ antennas indicates a much sharper drop for wider beamwidth antennas. Initially, this may not seem intuitive, but since the $60^{\circ} \mathrm{HPBW}$ sectored antennas have a wider viewing angle, their mean attenuations may not be as significant as for narrower beamwidth antennas such that a drop to the mean signal attenuation occurs at a faster rate. Additional studies are needed to support this conjecture.

The signal strength rise time $\left(r_{\text {rise }}\right)$ CDFs in Fig. 9b show that wider beamwidth antennas result in faster rise time rates than narrowbeam antennas. For instance, the $15^{\circ}$ and $60^{\circ}$ HPBW TX/RX antenna pairs resulted in $0.13 \mathrm{~dB} / \mathrm{ms}$ and 0.14 $\mathrm{dB} / \mathrm{ms}$ median rising signal strength rates compared to a 0.10 $\mathrm{dB} / \mathrm{ms}$ increasing signal strength rate for the $7^{\circ} \mathrm{HPBW} \mathrm{TX} / \mathrm{RX}$ antennas. A noteworthy observation is that the decay $\left(r_{\text {decay }}\right)$ and rise $\left(r_{\text {rise }}\right)$ time rates are asymmetric, which was also noticed in [17]. The exact reason for this is unknown, but perhaps is an effect of the trajectories with which blockers cross the LOS path between the TX and RX antennas. Table IV gives the CDF parameters for the best-fit curves to empirical data in Fig. 9b along with high GOF values.

The mean signal attenuation for the three blockage tests resulted in an expected trend where the narrower beamwidth antennas demonstrated greater mean signal attenuations for

TABLE IV: Best-fit CDF parameters of the Four-State Markov models for the $73.5 \mathrm{GHz}$ rapid fading events caused by pedestrian traffic.

\begin{tabular}{|c|c|c|c|}
\hline \multicolumn{4}{|c|}{ CDF Parameters for Signal Strength Decay Rate: $\boldsymbol{r}_{\text {decay }}[\mathbf{d B} / \mathbf{m s}]$} \\
\hline Ant. HPBW & Dist. & Parameters & GOF \\
\hline $7^{\circ}$ & Log-normal & $\mu=-2.15 ; \sigma=0.43$ & 0.96 \\
\hline $15^{\circ}$ & Log-normal & $\mu=-2.07 ; \sigma=0.61$ & 0.98 \\
\hline $60^{\circ}$ & Log-normal & $\mu=-1.87 ; \sigma=0.51$ & 0.98 \\
\hline \multicolumn{4}{|c|}{ CDF Parameters for Signal Strength Rise Rate: $\boldsymbol{r}_{\text {rise }}[\mathbf{d B} / \mathbf{m s}]$} \\
\hline Ant. HPBW & Distribution & Parameters & GOF \\
\hline $7^{\circ}$ & Log-normal & $\mu=-2.22 ; \sigma=0.39$ & 0.99 \\
\hline $15^{\circ}$ & Log-normal & $\mu=-2.17 ; \sigma=0.55$ & 0.99 \\
\hline $60^{\circ}$ & Log-normal & $\mu=-1.95 ; \sigma=0.56$ & 0.95 \\
\hline CDF Parameters for Shadowing Event Mean Attenuation: $\boldsymbol{S E}$ mean $[\mathbf{d B}]$ \\
\hline Ant. HPBW & Distribution & Parameters & GOF \\
\hline $7^{\circ}$ & Log-normal & $\mu=2.71 ; \sigma=0.31$ & 0.97 \\
\hline $15^{\circ}$ & Uniform & $A=5.11 ; B=19.02$ & 0.98 \\
\hline $60^{\circ}$ & Normal & $\mu=11.50 ; \sigma=2.41$ & 0.96 \\
\hline \multicolumn{4}{|c|}{ CDF Parameters for Fade Time Duration: $\boldsymbol{t}_{\boldsymbol{D}}[\mathbf{m s}]$} \\
\hline Ant. HPBW & Distribution & Parameters & GOF \\
\hline $7^{\circ}$ & Log-normal & $\mu=5.87 ; \sigma=0.35$ & 0.94 \\
\hline $15^{\circ}$ & Log-normal & $\mu=5.69 ; \sigma=0.53$ & 0.93 \\
\hline $60^{\circ}$ & Log-normal & $\mu=5.58 ; \sigma=0.49$ & 0.95 \\
\hline
\end{tabular}


individual blockage events, as shown by the CDFs in Fig. 9c (See Table IV for parameters). Specifically, the $7^{\circ} \mathrm{HPBW}$ antennas produced an average signal attenuation of $15.8 \mathrm{~dB}$ whereas the $15^{\circ}$ and $60^{\circ} \mathrm{HPBW}$ antennas demonstrated an average signal fade of $12.4 \mathrm{~dB}$ and $11.5 \mathrm{~dB}$, respectively. The inverse relationship between antenna HPBW and mean signal attenuation can be attributed to the larger beamwidth antennas capturing more diffracted energy around the human blockers during the blockage event, compared to narrower beam antennas that act as spatial filters.

A simple analytical expression may be used to determine the mean signal attenuation as a function of the TX/RX antenna HPBW:

$$
\text { Mean Blockage Attenuation }[\mathrm{dB}]=10 \log _{10}\left(b+\frac{180}{A_{B W}}\right)
$$

where $b$ is a constant and $A_{B W}$ is the TX/RX antenna HPBW in degrees. Fig. 10 shows all mean attenuations for each TX/RX HPBW antenna pair and the average mean attenuation for each pair. A rough estimate for the model in (2) is fit to the average mean signal attenuations in Fig. 10 , with $b=9.8$. The model is used to describe the observation of the physical environment where a single LOS cluster is blocked and when no secondary paths are available. As the TX/RX antenna HPBW increases, the mean signal attenuation decreases since a larger and equal spread of the wavefront impacts the blocker leading to more energy diffracted around the blocker and thus captured by a wider viewing angle at the RX. For very narrow HPBW antennas, the mean signal attenuation will be large since the spread of the transmitted wavefront and viewing angle at the receiver are fully blocked by an obstruction, leading to little-diffracted energy observed at the RX. An interesting note is that there is a limit at which increasing the antenna HPBW will have inconsequential benefits since the LOS path cluster takes such a narrow trajectory between the TX and RX in absence of secondary reflectors and scatterers. As shown in Fig. 10, the minimum mean signal attenuation reaches a limit of approximately $10 \mathrm{~dB}$ for TX/RX antenna HPBWs of $70^{\circ}$ or more. Since only 3 data points were used to model (2), future work will be done to further verify the model.

Fade time durations $\left(t_{D}\right)$ are shown by the CDFs in Fig. $9 \mathrm{~d}$, where on average the wider beamwidth antennas demonstrated shorter fade durations than narrower beam antennas. Parameters

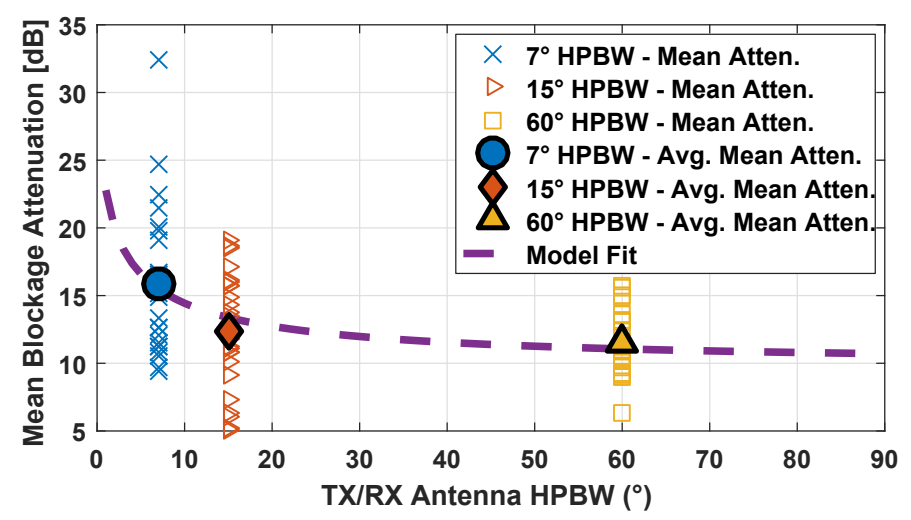

Fig. 10: Model fit for (2) showing that mean blockage attenuation is inversely related to the TX/RX antenna HPBW.
TABLE V: Four-state Markov model transition probability rates for human blockage events at $73.5 \mathrm{GHz}$

\begin{tabular}{|c|c|c|c|}
\hline TX/RX HPBW & Current State & Next State & $\lambda$ - rate (trans/sec) \\
\hline \multirow{4}{*}{$7^{\circ}$} & unshadowed & decaying & 0.21 \\
\cline { 2 - 4 } & decaying & shadowed & 7.88 \\
\cline { 2 - 4 } & shadowed & rising & 7.70 \\
\cline { 2 - 4 } & rising & unshadowed & 7.67 \\
\hline \multirow{4}{*}{$15^{\circ}$} & unshadowed & decaying & 0.21 \\
\cline { 2 - 4 } & decaying & shadowed & 10.49 \\
\cline { 2 - 4 } & shadowed & rising & 9.79 \\
\cline { 2 - 4 } & rising & unshadowed & 5.48 \\
\hline \multirow{5}{*}{$60^{\circ}$} & unshadowed & decaying & 0.18 \\
\cline { 2 - 4 } & decaying & shadowed & 11.30 \\
\cline { 2 - 4 } & shadowed & rising & 10.36 \\
\cline { 2 - 4 } & rising & unshadowed & 6.88 \\
\hline
\end{tabular}

corresponding to the CDF curves best-fit to the measurement data are provided in Table IV. The larger fade time durations for narrowbeam antennas compared to widebeam antennas is expected since wider beamwidth antennas have larger viewing angles to capture diffracted energy around a pedestrian blocker. Specifically, the $60^{\circ} \mathrm{HPBW}$ antennas resulted in a mean fade time duration of $321.3 \mathrm{~ms}$, whereas the $7^{\circ} \mathrm{HPBW}$ antennas demonstrated mean fade durations of $378.0 \mathrm{~ms}$. The larger fade time durations for the narrowbeam antennas might give an indication as to why the signal strength decay and rise rates are slower than for wider beamwidth antennas as observed earlier. Note that the fade time durations for the four-state model are longer than the two-state model since the definitions and thresholds are slightly different in each case (see Sections III-A and III-B).

Since the durations of fading events are on the order of 100 's of milliseconds, mmWave systems must be designed to electrically switch beams on the order of microseconds to find an unblocked reflector or to use TX diversity through a distributed multiple-input and multiple-output (MIMO) architecture where multiple nodes can serve the same user. More specifically, it may be required to implement double-link beam tracking algorithms [27] in lieu of single-link beam tracking which is currently implemented in the 802.11ad standard.

The four-state Markov model transition probability rates are provided in Table $\mathrm{V}$ and are useful for creating simple simulations of human blockage events for various antenna HPBW configurations. The CDF parameters provided in Table IV are also useful for designing PHY and MAC layer protocols, beam switching and beam steering algorithms, or for modeling performance of small cell networks at mmWave frequencies.

\section{CONCLUSION}

In this paper, we have demonstrated a mmWave measurement campaign conducted at $73.5 \mathrm{GHz}$ and with $1 \mathrm{GHz}$ of RF bandwidth to study the effects of pedestrian foot-traffic on a peer-topeer / point-to-point mmWave link in a busy urban scenario. Two simple blockage event models were described from the literature where one used a pre-defined threshold to determine the shadowed and unshadowed states and the second approach models four blockage event states: unshadowed, decaying signal strength, shadowed, and increasing signal strength. The two-state Markov model indicates that shadowing events are longer when using narrowbeam antennas compared to widebeam antennas. 
The conditional transition probability rates given in Table II can be used to simulate random blockage events in system and network level simulators for mmWave systems [6].

The four-state piecewise linear blockage model indicates the rate of decaying signal strength and rising signal strength at the beginning and end of each blockage event by the CDF plots shown in Section IV-B, where the rates are asymmetrical for the blockage events. The decay and rise rates are likely asymmetrical due to non-symmetrical blockers that caused signal fades when multiple people walked together, or by crossing the LOS path between the TX and RX antennas at nonperpendicular trajectories. The CDFs of the mean signal fade attenuations for the three antenna HPBW pairs demonstrate that average signal fades are inversely related to antenna HPBW such that the $60^{\circ}, 15^{\circ}$, and $7^{\circ} \mathrm{TX} / \mathrm{RX}$ HPBW antenna pairs had monotonically increasing mean signal attenuations of 11.5 $\mathrm{dB}, 12.4 \mathrm{~dB}$, and $15.8 \mathrm{~dB}$, respectively. A simple model for mean signal attenuation as a function of antenna HPBW is introduced for this physical observation. The observation that fade depth is a function of antenna beamwidth is similar to other work that showed path loss, delay spread, and azimuth spreads are functions of antenna beamwidth and frequency [25]. Furthermore, previous studies reported similar observations with mean signal attenuations between $6 \mathrm{~dB}$ and $18 \mathrm{~dB}$ [11] at 60 GHz. On average, blockage events were longer for narrower beamwidth antennas since they have a much smaller viewing angle to capture energy as compared to wider HPBW antennas, as shown by the $378.0 \mathrm{~ms}$ mean fade time duration for $7^{\circ}$ HPBW antennas compared to $321.3 \mathrm{~ms}$ for $60^{\circ} \mathrm{HPBW}$ antennas. A remarkable result is that the median fade time durations are approximately $255 \mathrm{~ms}$ and $327 \mathrm{~ms}$ for the two-state and four-state models, respectively, no matter what the TX and RX antenna beamwidths are, which may be a result of only detecting a LOS path cluster over all measurements. The fade durations reported here are in agreement with earlier studies at $60 \mathrm{GHz}$ that were on the order of $300 \mathrm{~ms}$ to $500 \mathrm{~ms}$ [10], [11].

The blockage events observed here for peer-to-peer networks in a dense urban crowded area are important for designing mmWave communications systems that rely on electrically steerable antenna systems to consistently deliver seamless connectivity and high-throughput in the presence of pedestrians and rapid fading. As the technology for electrically-steerable and reconfigurable antenna arrays matures, future studies will be necessary for understanding the performance of switching antenna beams at microsecond speeds to find reflectors and scatterers to maintain sufficient SNR when the dominant path between the TX and RX is heavily obstructed.

\section{REFERENCES}

[1] T. S. Rappaport et al., "Millimeter Wave Mobile Communications for 5G Cellular: It Will Work!" IEEE Access, vol. 1, pp. 335-349, May 2013.

[2] F. Boccardi et al., "Five disruptive technology directions for 5G," IEEE Communications Magazine, vol. 52, no. 2, pp. 74-80, Feb. 2014.

[3] 3GPP, "Technical specification group radio access network; study on channel model for frequencies from 0.5 to $100 \mathrm{GHz}$ (Release 14)," 3rd Generation Partnership Project (3GPP), TR 38.901 V1.0.1, Mar. 2017.

[4] Aalto University, AT\&T, BUPT, CMCC, Ericsson, Huawei, Intel, KT Corporation, Nokia, NTT DOCOMO, New York University, Qualcomm, Samsung, University of Bristol, and University of Southern California, "5G channel model for bands up to $100 \mathrm{GHz}, "$ 2016, Oct. 21. [Online]. Available: http://www.5gworkshops.com/5GCM.html
[5] S. Sun et al., "Investigation of prediction accuracy, sensitivity, and parameter stability of large-scale propagation path loss models for 5G wireless communications (Invited Paper)," IEEE Transactions on Vehicular Technology, vol. 65, no. 5, pp. 2843-2860, May 2016.

[6] S. Sun, G. R. MacCartney, Jr., and T. S. Rappaport, "A novel millimeterwave channel simulator and applications for $5 \mathrm{G}$ wireless communications," in 2017 IEEE International Conference on Communications (ICC), May 2017, pp. 1-7.

[7] S. Sun et al., "Millimeter wave small-scale spatial statistics in an urban microcell scenario," in 2017 IEEE International Conference on Communications (ICC), May 2017, pp. 1-7.

[8] P. A. Eliasi and S. Rangan, "Stochastic dynamic channel models for millimeter cellular systems," in 2015 IEEE 6th International Workshop on Computational Advances in Multi-Sensor Adaptive Processing (CAMSAP), Dec. 2015, pp. 209-212.

[9] M. Zhang et al., "Transport layer performance in $5 \mathrm{G}$ mmWave cellular," in 2016 IEEE Conference on Computer Communications Workshops (INFOCOM WKSHPS), Apr. 2016, pp. 730-735.

[10] S. Collonge, G. Zaharia, and G. El Zein, "Influence of the human activity on wide-band characteristics of the $60 \mathrm{GHz}$ indoor radio channel," IEEE Transactions on Wireless Communications, vol. 3, no. 6, pp. 2396-2406, Nov. 2004.

[11] M. Jacob, C. Mbianke, and T. Kürner, "A dynamic $60 \mathrm{GHz}$ radio channel model for system level simulations with MAC protocols for IEEE 802.11ad," in IEEE International Symposium on Consumer Electronics (ISCE 2010), June 2010, pp. 1-5.

[12] _ , "Human body blockage - guidelines for TGad MAC development," doc.: IEEE 802.11-09/1169r0, Nov. 2009.

[13] G. R. MacCartney, Jr. et al., "Millimeter-wave human blockage at 73 $\mathrm{GHz}$ with a simple double knife-edge diffraction model and extension for directional antennas," in 2016 IEEE 84th Vehicular Technology Conference (VTC2016-Fall), Sept. 2016, pp. 1-6.

[14] A. Maltsev et al., "Channel Models for $60 \mathrm{GHz}$ WLAN Systems," doc.: IEEE 802.11-09/0334r8, May 2010.

[15] R. J. Weiler et al., "Measuring the busy urban $60 \mathrm{GHz}$ outdoor access radio channel," in 2014 IEEE International Conference on Ultra-WideBand (ICUWB), Sept. 2014, pp. 166-170.

[16] _ , "Environment induced shadowing of urban millimeter-wave access links," IEEE Wireless Communications Letters, vol. 5, no. 4, pp. 440-443, Aug. 2016.

[17] M. Peter et al., "Analyzing human body shadowing at $60 \mathrm{GHz}$ : Systematic wideband MIMO measurements and modeling approaches," in 2012 6th European Conference on Antennas and Propagation (EuCAP), Mar. 2012, pp. $468-472$.

[18] J. Kunisch and J. Pamp, "Ultra-wideband double vertical knife-edge model for obstruction of a ray by a person," in 2008 IEEE International Conference on Ultra-Wideband, vol. 2, Sept. 2008, pp. 17-20.

[19] W. Q. Malik, B. Allen, and D. J. Edwards, "Impact of bandwidth on small-scale fade depth," in IEEE GLOBECOM 2007 - IEEE Global Telecommunications Conference, Nov. 2007, pp. 3837-3841.

[20] J. M. Holtzman and L. M. A. Jalloul, "Rayleigh fading effect reduction with wideband DS/CDMA signals," IEEE Transactions on Communications, vol. 42, no. 234, pp. 1012-1016, Feb. 1994.

[21] G. R. MacCartney, Jr. and T. S. Rappaport, "A flexible millimeter-wave channel sounder with absolute timing," IEEE Journal on Selected Areas in Communications, vol. 35, no. 6, pp. 1402-1418, June 2017.

[22] I. Kashiwagi, T. Taga, and T. Imai, "Time-varying path-shadowing model for indoor populated environments," IEEE Transactions on Vehicular Technology, vol. 59, no. 1, pp. 16-28, Jan. 2010.

[23] S. Dehnie, "Markov chain approximation of rayeleigh fading channel," in 2007 IEEE International Conference on Signal Processing and Communications, Nov. 2007, pp. 1311-1314.

[24] M. Jacob et al., "Modeling the dynamical human blockage for $60 \mathrm{GHz}$ WLAN channel models," in doc.: IEEE 802.11-10/0090r0, Jan. 2010.

[25] T. S. Rappaport et al., "Wideband millimeter-wave propagation measurements and channel models for future wireless communication system design (Invited Paper)," IEEE Transactions on Communications, vol. 63, no. 9, pp. 3029-3056, Sept. 2015.

[26] S. Singh et al., "Blockage and directivity in $60 \mathrm{GHz}$ wireless personal area networks: from cross-layer model to multihop MAC design," IEEE Journal on Selected Areas in Communications, vol. 27, no. 8, pp. 1400-1413, Oct. 2009.

[27] B. Gao et al., "Double-link beam tracking against human blockage and device mobility for 60-GHz WLAN," in 2014 IEEE Wireless Communications and Networking Conference (WCNC), Apr. 2014, pp. 323-328. 